\title{
A LINEAR APPROACH TO LIE TRIPLE AUTOMORPHISMS OF $H^{*}$-ALGEBRAS
}

\author{
A. J. Calderón Martín and C. Martín GonzÁlez
}

\begin{abstract}
By developing a linear algebra program involving many different structures associated to a three-graded $H^{*}$-algebra, it is shown that if $L$ is a Lie triple automorphism of an infinite-dimensional topologically simple associative $H^{*}$-algebra $A$, then $L$ is either an automorphism, an anti-automorphism, the negative of an automorphism or the negative of an anti-automorphism. If $A$ is finite-dimensional, then there exists an automorphism, an anti-automorphism, the negative of an automorphism or the negative of an anti-automorphism $F: A \rightarrow A$ such that $\delta:=F-L$ is a linear map from $A$ onto its center sending commutators to zero. We also describe $L$ in the case of having $A$ zero annihilator.
\end{abstract}

\section{Introduction}

Let $A$ be an associative algebra over the complex field $\mathbb{C}$. Then $A$ is a Lie algebra under the Lie multiplication $[a, b]=a b-b a$, and a Lie triple system under the triple product $[a, b, c]=[[a, b], c]$. A linear isomorphism $L: A \rightarrow A^{\prime}$ between two associative algebras is called a Lie triple isomorphism if $L([a, b, c])=[L(a), L(b), L(c)]$ for all $a, b, c \in A$. Isomorphisms, antiisomorphisms, negative of isomorphisms and negative of anti-isomorphisms $f: A \rightarrow A^{\prime}$ are examples of Lie triple isomorphisms. We also recall that a linear isomorphism $l: A \rightarrow A^{\prime}$ is said to be a Lie isomorphism if $l([a, b])=[l(a), l(b)]$ for any $a, b \in A$.

The structure of Lie isomorphisms has attracted some attention over past years: in the case of rings $[2,3,4,7,16,17]$, in the case of Banach algebras $[5,26,27]$ and in the case of $H^{*}$-algebras [11]. Obviously, every Lie isomorphism is a Lie triple isomorphism. The converse is, in general, not true. In the recent years, there also has been some interest in studying Lie triple isomorphisms

Received April 22, 2009.

2010 Mathematics Subject Classification. 17B40, 46L40, 47B47, 46K70.

Key words and phrases. $H^{*}$-algebra, graded algebra, Jordan pair, Lie triple automorphism.

Supported by the PCI of the UCA 'Teoría de Lie y Teoría de Espacios de Banach', by the PAI with project numbers FQM-298 and FQM-336, by the project of the Spanish Ministerio de Educación y Ciencia MTM2004-06580-C02-02 and with fondos FEDER. 
and derivations of different structures $[24,30]$. In this paper we consider Lie triple automorphisms of $H^{*}$-algebras.

Using functional identities one can describe Lie triple isomorphisms at a high level of generality, [6, Corollary 1]. However, we use entirely different methods to characterize Lie triple isomorphisms of $H^{*}$-algebras. In fact, we note that the development of a linear algebra program relating many different structures associated to a three-graded $H^{*}$-algebra, see Proposition 2.2, to the treatment of this problem is perhaps the most interesting novelty in this paper.

We recall that an $H^{*}$-algebra $A$ over $\mathbb{C}$ is a, non-necessarily associative, $\mathbb{C}$ algebra whose underlying vector space is a complex Hilbert space with inner product $(\cdot \mid \cdot)$, endowed with a conjugate-linear map $*: A \rightarrow A\left(x \mapsto x^{*}\right)$, such that $\left(x^{*}\right)^{*}=x,(x y)^{*}=y^{*} x^{*}$ for any $x, y \in A$ and the following identities, $\left(H^{*}\right.$-identities $)$, hold

$$
(x y \mid z)=\left(x \mid z y^{*}\right)=\left(y \mid x^{*} z\right)
$$

for all $x, y, z \in A$. The map $*$ will be called the involution of the $H^{*}$-algebra. The continuity of the product of $A$ is proved in [20]. We call the $H^{*}$-algebra $A$, topologically simple if $A^{2} \neq 0$ and $A$ has no nontrivial closed ideals. $H^{*}$-algebras were introduced and studied by Ambrose [1] in the associative case, and have been also considered in the case of the most familiar classes of nonassociative context $[8,18,20,29]$ and even in the general nonassociative context $[19,20]$. In [20] it is prove that any $H^{*}$-algebra $A$ with continuous involution splits into the orthogonal direct sum $A=\operatorname{Ann}(A) \perp \overline{\mathcal{L}\left(A^{2}\right)}$, where $\operatorname{Ann}(A):=\{x \in A: x A=$ $A x=0\}$ is the annihilator of $A$, and $\overline{\mathcal{L}\left(A^{2}\right)}$ is the closure of the vector span of $A^{2}$, which turns out to be an $H^{*}$-algebra with zero annihilator. Moreover, each $H^{*}$-algebra $A$ with zero annihilator satisfies $A=\overline{\perp I_{\alpha}}$, where $\left\{I_{\alpha}\right\}_{\alpha}$ denotes the family of minimal closed ideals of $A$, each of them being a topologically simple $H^{*}$-algebra. We also recall that any isomorphism $f$ on arbitrary $H^{*}$-algebras with zero annihilator is continuous and that if $f$ commutes with the involution, (we say that $f$ is an $*$-isomorphism), and the $H^{*}$-algebras are topologically simple, then $f$ is isometric up to a positive multiple of the inner product [19].

$H^{*}$-triple systems appears as the ternary version of $H^{*}$-algebras. It is well known that $H^{*}$-triple systems and their isomorphisms have similar properties to those of $H^{*}$-algebras described above (see $[15,14]$ ).

Let $H$ be a complex Hilbert space and let $\mathcal{H} S(H)$ be the algebra of all Hilbert-Schmidt operators on $H$. If $\left\{\phi_{i}\right\}_{i \in \mathcal{A}}$ is a complete orthonormal system of $H$ and $f, g \in \mathcal{H} S(H)$, then the sum $\sum\left(f\left(\phi_{i}\right) \mid g\left(\phi_{i}\right)\right)$ is independent of the choice of $\left\{\phi_{i}\right\}_{i \in \mathcal{A}}$. It can be proved that $\mathcal{H} S(H)$ becomes an associative $H^{*}$ algebra under the inner product

$$
(f \mid g)=\sum\left(f\left(\phi_{i}\right) \mid g\left(\phi_{i}\right)\right)
$$

and the involution $f \mapsto f^{*}$, where $f^{*}$ is the adjoint operator of $f$, that is, the unique element in $\mathcal{H} S(H)$ such that $(f(x) \mid y)=\left(x \mid f^{*}(y)\right)$ for all $x, y \in H$. It is prove in [1] that any infinite dimensional topologically simple associative 
$H^{*}$-algebra is $*$-isometrically isomorphic (up to a positive multiple of the inner product) to $\mathcal{H} S(H)$ with $H$ a complex Hilbert space of infinite hilbertian dimension. We can give a matrix expression of topologically simple associative $H^{*}$-algebras as follows: In the infinite-dimensional case, if $A$ is a non-empty set, we denote by $\mathcal{M}_{A}(\mathbb{C})$ the associative topologically simple complex $H^{*}$ algebra of all matrices $B=\left(b_{i, j}\right)_{i, j \in A}$ with elements in $\mathbb{C}$ such that $\sum\left\|b_{i, j}\right\|^{2}$ converges. The inner product is $\left(\left(a_{i, j}\right) \mid\left(b_{i, j}\right)\right)=\sum a_{i, j} \overline{b_{i, j}}$ and the involution $\left(a_{i, j}\right)^{*}=\left(\overline{a_{j, i}}\right)$. Fixed $B=\left\{\phi_{i}\right\}_{i \in A}$ a complete orthonormal system of a complex Hilbert space $H$, if $\left\{E_{i, j}\right\}_{(i, j) \in A \times A}$ is the family of the operators of $\mathcal{H} S(H)$ defined by $E_{i, j}\left(\phi_{k}\right)=\delta_{j, k} \phi_{i}$ (where $\delta_{j, k}$ is the Kronecker delta), then the continuous linear mapping

$$
\begin{aligned}
\Phi: \mathcal{H} S(H) & \rightarrow \mathcal{M}_{A}(\mathbb{C}) \\
E_{i, j} & \mapsto e_{i, j}
\end{aligned}
$$

where $e_{i, j}$ denotes the usual elemental matrix, is an isometric $*$-isomorphism of associative $H^{*}$-algebras. We also recall that any simple associative $H^{*}$-algebra $A$ with finite dimension is isometrically $*$-isomorphic (up to a positive multiple of the inner product) to an $H^{*}$-algebra of the type $\mathcal{M}_{n}(\mathbb{C})$.

The paper is organized as follows. In Section 2 we introduce the different $H^{*}$-structures associated to an associative three-graded $H^{*}$-algebra, and study the transference of the topological simplicity among them. These results will make us easier the development of the following section. Finally, we study in Section 3 the Lie triple automorphisms of topologically simple associative $H^{*}$-algebras and of associative $H^{*}$-algebras with zero annihilator.

\section{Different structures on $\boldsymbol{H}^{*}$-algebras}

Let $K$ be a unitary commutative ring. A three-graded $K$-algebra $A$ is a $K$-algebra which splits into the direct sum $A=A_{-1} \oplus A_{0} \oplus A_{1}$ of nonzero $K$-submodules satisfying $A_{0} A_{i}+A_{i} A_{0} \subset A_{i}$ for all $i \in\{-1,0,1\}$,

$$
A_{-1} A_{1}+A_{1} A_{-1} \subset A_{0}
$$

and $A_{1} A_{1}=A_{-1} A_{-1}=0$. A three-graded $H^{*}$-algebra is an $H^{*}$-algebra which is a three-graded algebra such that $A_{-1}, A_{0}, A_{1}$ are closed orthogonal subspaces satisfying $\left(A_{i}\right)^{*}=A_{-i}$ for $i \in\{-1,0,1\}$. We note that a topologically simple associative $H^{*}$-algebra can be three-graded in several different ways (see the proof of Theorem 3.1).

The following result, of easy verification, is proved in [11, Proposition 2.2].

Proposition 2.1. Let $A$ be an $H^{*}$-algebra with zero annihilator, and let $D$ be a self-adjoint derivation on $A$ with minimal polynomial $x^{3}-x$ and satisfying * $\circ D=-D \circ *$. Then $A=A_{-1} \perp A_{0} \perp A_{1}$ with $A_{i}=\operatorname{Ker}(D-i I d)$, $i \in\{-1,0,1\}$, is a three-graded $H^{*}$-algebra.

Lemma 2.1. Let $A$ and $D$ be as in Proposition 2.1. Then

$$
\operatorname{Ker}(D+I d)+\operatorname{Ker}(D-I d)=\operatorname{Ker}\left(D^{2}-I d\right) .
$$


Proof. The inclusion $\operatorname{Ker}(D+I d)+\operatorname{Ker}(D-I d) \subset \operatorname{Ker}\left(D^{2}-I d\right)$ is clear. Conversely, if $x \in \operatorname{Ker}\left(D^{2}-I d\right)$, by Proposition 2.1 we can write $x=x_{-1}+x_{0}+$ $x_{1}$ with $x_{i} \in \operatorname{Ker}(D-i I d), i \in\{-1,0,1\}$. As $x=D^{2}(x)=D D\left(x_{-1}+x_{0}+x_{1}\right)=$ $D\left(-x_{-1}+x_{1}\right)=x_{-1}+x_{1}$, we finally obtain $x \in \operatorname{Ker}(D+I d)+\operatorname{Ker}(D-I d)$.

Let $A$ be a complex vector space. We say that $A$ is a ternary algebra if it is endowed with a trilinear map $\langle\cdot, \cdot, \cdot\rangle: A \times A \times A \rightarrow A$, called the triple product, satisfying

$$
\langle\langle x, y, z\rangle, u, v\rangle=\langle x,\langle y, z, u\rangle, v\rangle=\langle x, y,\langle z, u, v\rangle\rangle
$$

for any $x, y, z, u, v \in A$. If $A$ is an associative algebra, then the underlying vector space of $A$ with the triple product $\langle x, y, z\rangle:=x y z$ is a ternary algebra. We denote it $\mathcal{T} \mathcal{A}(A)$. A ternary $H^{*}$-algebra $T$ is a ternary algebra with an involution $*$ (involutive conjugate-linear map $*: A \rightarrow A$ such that $\langle x, y, z\rangle^{*}=$ $\left.\left\langle z^{*}, y^{*}, x^{*}\right\rangle\right)$, and a Hilbert space structure with inner product $(\cdot \mid \cdot)$, satisfying

$$
(\langle x, y, z\rangle \mid u)=\left(x \mid\left\langle u, z^{*}, y^{*}\right\rangle\right)=\left(y \mid\left\langle x^{*}, u, z^{*}\right\rangle\right)=\left(z \mid\left\langle y^{*} x^{*}, u\right\rangle\right)
$$

for any $x, y, z, u \in T$. If $A$ is an associative $H^{*}$-algebra, then $\mathcal{T} \mathcal{A}(A)$ with the same involution and inner product than $A$ is a ternary $H^{*}$-algebra.

Let us construct some $H^{*}$-structures associated to an associative threegraded $H^{*}$-algebra. Let $A=A_{-1} \perp A_{0} \perp A_{1}$ be an associative three-graded $H^{*}$-algebra. Then $\mathcal{A P}(A):=\left(A_{-1}, A_{1}\right)$ and $\mathcal{P} \mathcal{T} \mathcal{A}(A):=A_{-1} \perp A_{1}$ are, respectively, an associative $H^{*}$-pair with the triple products $\left\langle a^{\sigma}, b^{-\sigma}, c^{\sigma}\right\rangle:=a^{\sigma} b^{-\sigma} c^{\sigma}$ for any $a^{\sigma}, c^{\sigma} \in A_{\sigma}, b^{-\sigma} \in A_{-\sigma}$; and a polarized ternary $H^{*}$-algebra with the triple product $\left\langle\left(a^{1}, a^{-1}\right),\left(b^{1}, b^{-1}\right),\left(c^{1}, c^{-1}\right)\right\rangle:=\left(a^{1} b^{-1} c^{1}, a^{-1} b^{1} c^{-1}\right)$ for any $a^{\sigma}, b^{\sigma}, c^{\sigma} \in A_{\sigma}$, and with the involutions and inner products induced by the ones in $A$. See $[9,12,15]$ for the definitions and first results on associative $H^{*}$-pairs and polarized ternary $H^{*}$-algebras.

We have that $A$ with the Lie bracket $[a, b]=a b-b a$ and with the triple product

$$
[a, b, c]=[[a, b] c]
$$

(and the same involution and inner product), becomes respectively a Lie $H^{*}$ algebra, (or $L^{*}$-algebra), and a Lie $H^{*}$-triple system, (or $L^{*}$-triple), that we denote by $A^{-}$and $\mathcal{L} \mathcal{T}(A)$ resp. We also can check that

$$
\mathcal{Z}_{2} \mathcal{L}(A):=A_{0} \perp\left(A_{-1}+A_{1}\right)
$$

with the Lie bracket is a $\mathbb{Z}_{2}$-graded $L^{*}$-algebra, and that $\mathcal{P} \mathcal{L} \mathcal{T}(A):=A_{-1} \perp A_{1}$ is a polarized $L^{*}$-triple with the triple product (1) (and with the involutions and inner products induced by $A)$. See $[29,18,10]$ for the definitions and basic results on $L^{*}$-algebras, $L^{*}$-triples and $\mathbb{Z}_{2}$-graded $L^{*}$-algebras. See also $[13,28]$ for the definition of polarized $L^{*}$-triple.

Finally, we have that $\mathcal{J P}(A):=\left(A_{-1}, A_{1}\right)$ and $\mathcal{P} \mathcal{J} \mathcal{T}(A):=A_{-1} \perp A_{1}$ are, respectively, a Jordan $H^{*}$-pair with the quadratic operators $Q^{\sigma}\left(a^{\sigma}\right)\left(b^{-\sigma}\right)=$ $a^{\sigma} b^{-\sigma} a^{\sigma}$ for any $a^{\sigma} \in A_{\sigma}, b^{-\sigma} \in A_{-\sigma}$; and a polarized Jordan $H^{*}$-triple system with the quadratic operator $Q^{\sigma}\left(\left(a^{1}, a^{-1}\right)\right)\left(\left(b^{1}, b^{-1}\right)\right)=\left(a^{1} b^{-1} a^{1}, a^{-1} b^{1} a^{-1}\right)$ for 
all $a^{\sigma}, b^{\sigma} \in A_{\sigma}$, the involutions and inner products being the ones induced by $A$. See $[9,12,13]$ for the definitions and preliminary results on Jordan $H^{*}$-pairs and polarized Jordan $H^{*}$-triple systems.

Proposition 2.2. Let $A=A_{-1} \perp A_{0} \perp A_{1}$ be an infinite dimensional threegraded associative $H^{*}$-algebra. Then the following assertions are equivalent:

(1) A is topologically simple.

(2) The ternary $H^{*}$-algebra $\mathcal{T} \mathcal{A}(A)$ is topologically simple and $A_{0}=\overline{A_{-1} A_{1}+A_{1} A_{-1}}$.

(3) The associative $H^{*}$-pair $\mathcal{A P}(A)=\left(A_{-1}, A_{1}\right)$ is topologically simple and $A_{0}=\overline{A_{-1} A_{1}+A_{1} A_{-1}}$.

(4) The polarized ternary $H^{*}$-algebra $\mathcal{P} \mathcal{T} \mathcal{A}(A)=A_{-1} \perp A_{1}$ is topologically simple and $A_{0}=\overline{A_{-1} A_{1}+A_{1} A_{-1}}$.

(5) The $L^{*}$-algebra $A^{-}$is topologically simple.

(6) The $L^{*}$-triple $\mathcal{L} \mathcal{T}(A)$ is topologically simple.

(7) The $\mathbb{Z}_{2}$-graded $L^{*}$-algebra $\mathcal{Z}_{2} \mathcal{L}(A)=A_{0} \perp\left(A_{-1}+A_{1}\right)$ is topologically simple in graded sense and $A_{0}=\overline{A_{-1} A_{1}+A_{1} A_{-1}}$.

(8) The polarized $L^{*}$-triple $\mathcal{P} \mathcal{L} \mathcal{T}(A)=A_{-1} \perp A_{1}$ is topologically simple and $A_{0}=\overline{A_{-1} A_{1}+A_{1} A_{-1}}$.

(9) The Jordan $H^{*}$-pair $\mathcal{J P}(A)=\left(A_{-1}, A_{1}\right)$ is topologically simple and $A_{0}=\overline{A_{-1} A_{1}+A_{1} A_{-1}}$.

(10) The polarized Jordan $H^{*}$-triple system $\mathcal{P} \mathcal{J} \mathcal{T}(A)=A_{-1} \perp A_{1}$ is topologically simple and $A_{0}=\overline{A_{-1} A_{1}+A_{1} A_{-1}}$.

Proof. $(1) \Rightarrow(2)$. By $\S 1$, we can write $A=\mathcal{H} \mathcal{S}(H)$ and so, by the classification of topologically simple ternary $H^{*}$-algebras in [15, Main Theorem], we deduce $\mathcal{T} \mathcal{A}(A)$ is also topologically simple. By the continuity of the product, $A_{-1} \perp \overline{A_{-1} A_{1}+A_{1} A_{-1}} \perp A_{1}$ is a nonzero closed ideal of $A$ and so $A_{0}=\overline{A_{-1} A_{1}+A_{-1} A_{1}}$.

$(2) \Rightarrow(3)$. Let $\left(I_{-1}, I_{1}\right)$ be a nonzero closed ideal of $\mathcal{A P}(A)=\left(A_{-1}, A_{1}\right)$. As $I:=I_{-1} \perp \overline{\left(I_{-1} A_{1}+A_{-1} I_{1}+I_{1} A_{-1}+A_{1} I_{-1}\right)} \perp I_{1}$ is a nonzero closed ideal of $\mathcal{T} \mathcal{A}(A)$, then $I=\mathcal{T} \mathcal{A}(A)$ and so $\left(I_{-1}, I_{1}\right)=\left(A_{-1}, A_{1}\right)$.

$(3) \Rightarrow(4)$. Let $I$ be a nonzero closed ideal of $\mathcal{P} \mathcal{T} \mathcal{A}(A)$. If we denote by $\pi_{i}(I)$, $i \in \pm 1$ the orthogonal projections of $I$ over $A_{i}, i \in \pm 1$, it is easy to check that $\left(\pi_{-1}(I), \pi_{1}(I)\right)$ is a closed ideal of $\mathcal{A P}(A)$. Since $I \neq 0, \pi_{i}(I)=A_{i}$. Therefore, for any $x_{-1} \in A_{-1}$, there exists $x_{1} \in A_{1}$ such that $x_{-1}+x_{1} \in I$. Given now any $a_{-1} \in A_{-1}$ and $a_{1} \in A_{1}$ we have $\left(x_{-1}+x_{1}\right) a_{1} a_{-1}=x_{-1} a_{1} a_{-1} \in I \cap A_{-1}$. If $I \cap A_{-1}=0$, then $A_{-1} A_{1} A_{-1}=0$, contradicting the topological simplicity of $\mathcal{A P}(A)$. As $\left(I \cap A_{-1}, I \cap A_{1}\right)$ is an ideal of $\mathcal{A P}(A)$, we conclude $I \cap A_{i}=A_{i}$ and therefore $I=A_{-1}+A_{1}=\mathcal{P} \mathcal{T} \mathcal{A}(A)$.

$(4) \Rightarrow(1)$. By the classification of topologically simple ternary $H^{*}$-algebras [15], the $\mathbb{Z}_{2}$-graded envelope $H^{*}$-algebra of $\mathcal{P} \mathcal{T} \mathcal{A}(A)$ is topologically simple. As $A_{0}=\overline{A_{-1} A_{1}+A_{1} A_{-1}}$, we have that $A$ is the $\mathbb{Z}_{2}$-graded envelope $H^{*}$-algebra of $\mathcal{P} \mathcal{T} \mathcal{A}(A)$ and so it is topologically simple. 
$(1) \Leftrightarrow(5)$. It follows from the classifications of topologically simple associative and Lie $H^{*}$-algebras given in [20] and [18].

$(5) \Rightarrow(6) . \mathcal{L} \mathcal{T}(A)$ has $A \perp A$ as a $\mathbb{Z}_{2}$-graded envelope $L^{*}$-algebra. As $A \perp A$ is topologically simple in graded sense, then $\mathcal{L} \mathcal{T}(A)$ is topologically simple.

$(6) \Rightarrow(5)$. It is consequence of the fact that any ideal of $A^{-}$is also an ideal of $\mathcal{L} \mathcal{T}(A)$.

$(5) \Rightarrow(7)$. It is trivial.

$(7) \Leftrightarrow(8)$. It is well known that an $L^{*}$-triple is topologically simple if and only if its $\mathbb{Z}_{2}$-graded $L^{*}$-algebra envelope is topologically simple in graded sense [10]. From $A_{0}=\overline{A_{-1} A_{1}+A_{1} A_{-1}}$, we have $\mathcal{Z}_{2} \mathcal{L}(A)$ is a $\mathbb{Z}_{2}$-graded $L^{*}$-algebra envelope of $\mathcal{P} \mathcal{L} \mathcal{T}(A)$ and the equivalence is proved.

$(8) \Leftrightarrow(9) \Leftrightarrow(10)$. See $[13,28]$.

$(9) \Rightarrow(3)$. It is clear. See also [9, Theorem 2].

The following result can be proved as in [28] (see also [13, Proposition 3.2]).

Lemma 2.2. Let $V$ be a topologically simple Lie or Jordan $H^{*}$-triple system with two polarizations $V=V_{-1} \perp V_{1}$ and $V=W_{-1} \perp W_{1}$. Then either $V_{i}=W_{i}$ or $V_{i}=W_{-i}, i \in \pm 1$.

Lemma 2.3. Let $A=A_{-1} \perp A_{0} \perp A_{1}$ and $A^{\prime}=A_{-1}^{\prime} \perp A_{0}^{\prime} \perp A_{1}^{\prime}$ be two threegraded associative $H^{*}$-algebras. If $f: \mathcal{P} \mathcal{L} \mathcal{T}(A) \rightarrow \mathcal{P} \mathcal{L} \mathcal{T}\left(A^{\prime}\right)$ is a $*$-isomorphism of Lie triple systems, then $f: \mathcal{P} \mathcal{J} \mathcal{T}(A) \rightarrow \mathcal{P} \mathcal{J} \mathcal{T}\left(A^{\prime}\right)$ is a *-isomorphism of Jordan triple system.

Proof. It is immediate, taking into account that the quadratic operator of the polarized Jordan triple system $\mathcal{P} \mathcal{J} \mathcal{T}(A)$ can be written as

$$
Q\left(\left(x_{-1}, x_{1}\right)\right)\left(\left(y_{-1}, y_{1}\right)\right)=\left(\left[x_{-1}, y_{1}, x_{-1}\right],\left[x_{1}, y_{-1}, x_{1}\right]\right)
$$

where $[\cdot, \cdot, \cdot]$ denotes the triple product of $\mathcal{P} \mathcal{L} \mathcal{T}(A)$ (the same applies to $\left.\mathcal{P} \mathcal{J} \mathcal{T}\left(A^{\prime}\right)\right)$

\section{Lie triple automorphisms}

\subsection{The infinite dimensional case}

Theorem 3.1. Let $L$ be a Lie triple *-automorphism of an infinite dimensional topologically simple associative $H^{*}$-algebra $A$. Then, $A$ can be three-graded as $A=A_{-1} \perp A_{0} \perp A_{1}$ and as $A=A_{-1}^{\prime} \perp A_{0}^{\prime} \perp A_{1}^{\prime}$, in such a way that $A_{0}=$ $A_{00} \perp A_{01}$ and $A_{0}^{\prime}=A_{00}^{\prime} \perp A_{01}^{\prime}$ with $A_{00}, A_{01}, A_{00}^{\prime}, A_{01}^{\prime}$ topologically simple associative $H^{*}$-algebras, $A_{00}^{\prime}$ and $A_{00}^{\prime}$ of arbitrary finite dimension, and there exists an automorphism or an anti-automorphism $F$ of $A$ such that $F\left(A_{i}\right)=$ $L\left(A_{i}\right)=A_{i}^{\prime}, i \in \pm 1 ; F\left(A_{0 j}\right)=L\left(A_{0 j}\right)=A_{0 j}^{\prime}, j \in\{0,1\}$, and $F=L$ on $A_{-1} \perp A_{1}$.

Proof. First, let us three-graduate $A$ in two adequate ways. From $\S 1$, there is no loss of generality in writing $A=\mathcal{H} S(H)$, being $H$ a complex Hilbert space with infinite hilbertian dimension. As $L$ is a $*$-automorphism of the topologically 
simple $L^{*}$-triple $\mathcal{L} \mathcal{T}(A)$, we have that $L$ is isometric up to a positive multiple of the inner product (see $\S 1$ ). Let $\left\{\phi_{i}\right\}_{i \in \mathcal{A}}$ be a complete orthogonal system of $H$. We can express

$$
\left\{\phi_{i}\right\}_{i \in \mathcal{A}}=\left\{\phi_{i}\right\}_{i \in \mathcal{B}} \cup\left\{\phi_{i}\right\}_{i \in \mathcal{C}},
$$

being $\mathcal{B} \cup \mathcal{C}=\mathcal{A}, \mathcal{B} \cap \mathcal{C}=\emptyset, \mathcal{B}, \mathcal{C} \neq \emptyset$ and $\mathcal{B}$ a finite set. Consider the map $\operatorname{ad}(x): \mathcal{H} S(H) \rightarrow \mathcal{H} S(H)$, given by $\operatorname{ad}(x)(a)=[x, a]:=x a-a x$, being $x: H \rightarrow H$ defined by $x\left(\phi_{i}\right)=-\phi_{i}$ if $i \in \mathcal{B}$ and $x\left(\phi_{i}\right)=0$ if $i \in \mathcal{C}$. It is easy to prove that $\operatorname{ad}(x)$ is a self-adjoint derivation on $\mathcal{H} S(H)$ with minimal polynomial $x^{3}-x$ and satisfies

$$
* \circ \operatorname{ad}(x)=-\operatorname{ad}(x) \circ * .
$$

By Proposition 2.1,

$$
A=A_{-1} \perp A_{0} \perp A_{1}
$$

is a three-graded associative $H^{*}$-algebra, (topologically simple), being $A_{-1}=$ $\mathcal{H} S\left(H_{\mathcal{C}}, H_{\mathcal{B}}\right), A_{0}=\mathcal{H} S\left(H_{\mathcal{B}}\right) \perp \mathcal{H} S\left(H_{\mathcal{C}}\right)$, (we will write $A_{00}=\mathcal{H} S\left(H_{\mathcal{B}}\right), A_{01}=$ $\mathcal{H} S\left(H_{\mathcal{C}}\right)$ ); and $A_{1}=\mathcal{H} S\left(H_{\mathcal{B}}, H_{\mathcal{C}}\right)$, where $H_{\mathcal{B}}, H_{\mathcal{C}}$ denote the Hilbert subspaces of $H$ generated by $\left\{\phi_{i}\right\}_{i \in \mathcal{B}}$ and $\left\{\phi_{i}\right\}_{i \in \mathcal{C}}$ respectively, and $\mathcal{H} S\left(H_{\mathcal{C}}, H_{\mathcal{B}}\right)$ is the set of elements $g \in \mathcal{H} S(H)$ such that $g\left(H_{\mathcal{B}}\right)=0$ and $g\left(H_{\mathcal{C}}\right) \subset H_{\mathcal{B}}$ (the same applies to

$$
\mathcal{H} S\left(H_{\mathcal{B}}\right)=\mathcal{H} S\left(H_{\mathcal{B}}, H_{\mathcal{B}}\right), \quad \mathcal{H} S\left(H_{\mathcal{C}}\right)=\mathcal{H} S\left(H_{\mathcal{C}}, H_{\mathcal{C}}\right)
$$

and $\left.\mathcal{H} S\left(H_{\mathcal{B}}, H_{\mathcal{C}}\right)\right)$.

Let us show that $\operatorname{ad}(L(x))$ is a self-adjoint derivation of $A$ with minimal polynomial $x^{3}-x$ and such that $* \circ \operatorname{ad}(L(x))=-\operatorname{ad}(L(x)) \circ *$ : As $\operatorname{ad}(x)$ has minimal polynomial $x^{3}-x$, that is, $[x,[x,[x, y]]]=[x, y]$ for any $y \in A$, then $[[x,[x,[x, y]]], z]=[[x, y], z]$ for any $y, z \in A$. Therefore

$$
[[L(x),[L(x),[L(x), L(y)]]], L(z)]=[[L(x), L(y)], L(z)],
$$

that is,

$$
\left[\operatorname{ad}(L(x))^{3}(L(y)), L(z)\right]=[\operatorname{ad}(L(x))(L(y)), L(z)]
$$

for any $y, z \in A$ and so

$$
\left[\operatorname{ad}(L(x))^{3}(L(y))-\operatorname{ad}(L(x))(L(y)), L(z)\right]=0 .
$$

But $A^{-}$is topologically simple (see Proposition 2.2), and so $\operatorname{Ann}\left(A^{-}\right)=0$. Since $L$ is bijective, then $\operatorname{ad}(L(x))^{3}=\operatorname{ad}(L(x))$. A similar argument gives us $a d(L(x))^{2} \neq I d$ and so $a d(L(x))$ has minimal polynomial $x^{3}-x$. The fact that $* \circ \operatorname{ad}(L(x))=-\operatorname{ad}(L(x)) \circ *$ is a consequence of $x^{*}=x$, since for any $y \in A^{-}$we have $[L(x), y]^{*}=\left[y^{*}, L(x)^{*}\right]=\left[y^{*}, L\left(x^{*}\right)\right]=\left[y^{*}, L(x)\right]$. Finally, the self-adjoint character of $a d(L(x))$ easily follows from the $H^{*}$-identities in $A^{-}$and $L(x)^{*}=$ $L(x)$. By applying Proposition 2.1, we have that $A$ becomes a three-graded associative $H^{*}$-algebra as $A=A_{-1}^{\prime} \perp A_{0}^{\prime} \perp A_{1}^{\prime}$ with $A_{i}^{\prime}=\operatorname{Ker}(\operatorname{ad}(L(x))-i I d)$, $i \in\{-1,0,1\}$. To distinguish this new three-graduation of $A$, we will denoted $A^{\prime}:=A_{-1}^{\prime} \perp A_{0}^{\prime} \perp A_{1}^{\prime}$. 
Second, let us prove that $L\left(A_{i}\right)=A_{i}^{\prime}, i \in\{-1,0,1\}$. We begin by observing that the mapping $L$ is clearly a $*$-automorphism from

$$
\mathcal{L} \mathcal{T}(A)=\mathcal{L T}\left(A_{-1} \perp A_{0} \perp A_{1}\right)
$$

onto

$$
\mathcal{L} \mathcal{T}(A)=\mathcal{L} \mathcal{T}\left(A_{-1}^{\prime} \perp A_{0}^{\prime} \perp A_{1}^{\prime}\right) .
$$

Now, we can deduce $L\left(A_{-1} \perp A_{1}\right)=A_{-1}^{\prime} \perp A_{1}^{\prime}$, taking into account that given $a \in A_{i}, i \in \pm 1,[x,[x, a]]=a$. From here, $[L(x),[L(x), L(a)]]=L(a)$ and so

$$
L(a) \in \operatorname{Ker}\left(a d(L(x))^{2}-I d\right) .
$$

Lemma 2.1 gives now $L(a) \in A_{-1}^{\prime} \perp A_{1}^{\prime}$. Conversely, given $y \in A_{i}^{\prime}, i \in \pm 1$, a similar argument shows $L^{-1}(y) \in A_{-1} \perp A_{1}$, and then $L\left(A_{-1} \perp A_{1}\right)=$ $A_{-1}^{\prime} \perp A_{1}^{\prime}$. We now have that $L\left(A_{-1}\right) \perp L\left(A_{1}\right)$ is another polarization of $\mathcal{P} \mathcal{L} \mathcal{T}\left(A^{\prime}\right)=A_{-1}^{\prime} \perp A_{1}^{\prime}$, and as $\mathcal{P} \mathcal{L} \mathcal{T}\left(A^{\prime}\right)$ is topologically simple by Proposition 2.2, Lemma 2.2 shows either $L\left(A_{i}\right)=A_{i}^{\prime}$ or $L\left(A_{i}\right)=A_{-i}^{\prime}$. In the second case we define $A_{i}:=A_{-i}$. The isometric character (up to a positive multiple of the inner product) of $L$ implies $L\left(A_{0}\right)=A_{0}^{\prime}$.

Finally, let us prove the existence of $F$. We can apply Lemma 2.3 to conclude that $L: \mathcal{P} \mathcal{J} \mathcal{T}(A) \rightarrow \mathcal{P} \mathcal{J} \mathcal{T}\left(A^{\prime}\right)$ is a $*$-isomorphism of Jordan $H^{*}$-triple systems. We know that $\mathcal{P} \mathcal{J} \mathcal{T}(A)$ is a topologically simple polarized Jordan $H^{*}$-triple system coming from symmetrizing the topologically simple polarized ternary $H^{*}$-algebra $\mathcal{H} S\left(H_{\mathcal{C}}, H_{\mathcal{B}}\right) \perp \mathcal{H} S\left(H_{\mathcal{B}}, H_{\mathcal{C}}\right)$. Moreover, Proposition 2.2 gives us that $\mathcal{P} \mathcal{T} \mathcal{A}\left(A^{\prime}\right)=A_{-1}^{\prime} \perp A_{1}^{\prime}$ is a topologically simple polarized ternary $H^{*}$-algebra, and that $A_{0}^{\prime}=\overline{A_{-1}^{\prime} A_{1}^{\prime}+A_{1}^{\prime} A_{-1}^{\prime}}$. Since $\mathcal{P} \mathcal{J} \mathcal{T}\left(A^{\prime}\right)$ is then a topologically simple polarized Jordan $H^{*}$-triple system coming from symmetrizing a topologically simple polarized ternary $H^{*}$-algebra, the classifications of topologically simple polarized Jordan $H^{*}$-triple systems and ternary $H^{*}$-algebras in [12] and [15] give us that $\mathcal{P} \mathcal{J} \mathcal{T}\left(A^{\prime}\right)=\mathcal{H} S\left(H_{\mathcal{C}^{\prime}}^{\prime}, H_{\mathcal{B}^{\prime}}^{\prime}\right) \perp \mathcal{H} S\left(H_{\mathcal{B}^{\prime}}^{\prime}, H_{\mathcal{C}^{\prime}}^{\prime}\right)$ and that $A_{0}^{\prime}=A_{00}^{\prime} \perp A_{01}^{\prime}$ where $A_{00}^{\prime}=\mathcal{H} S\left(H_{\mathcal{B}^{\prime}}^{\prime}\right)$ and $A_{01}^{\prime}=\mathcal{H} S\left(H_{\mathcal{C}^{\prime}}^{\prime}\right)$; with $H_{\mathcal{B}^{\prime}}^{\prime}$ and $H_{\mathcal{C}^{\prime}}^{\prime}$ as above.

By applying now D'Amour's result ([21, Theorem B] or $[9$, Theorem 1]) as in the proof of [9, Theorem 2], $L: \mathcal{P} \mathcal{J} \mathcal{T}(A) \rightarrow \mathcal{P} \mathcal{J} \mathcal{T}(A)^{\prime}$ extends to an isomorphism of two-graded associative algebras

$$
\begin{gathered}
F: A \oplus A^{o p} \rightarrow A^{\prime} \oplus\left(A^{\prime}\right)^{o p}, \\
\text { being } A=\mathcal{H} S(H) \simeq\left(\begin{array}{cc}
\mathcal{H} S\left(H_{\mathcal{B}}\right) & \mathcal{H} S\left(H_{\mathcal{C}}, H_{\mathcal{B}}\right) \\
\mathcal{H} S\left(H_{\mathcal{B}}, H_{\mathcal{C}}\right) & \mathcal{H} S\left(H_{\mathcal{C}}\right)
\end{array}\right) \text { and } \\
A^{\prime}=\mathcal{H} S\left(H^{\prime}\right) \simeq\left(\begin{array}{cc}
\mathcal{H} S\left(H_{\mathcal{B}^{\prime}}^{\prime}\right) & \mathcal{H} S\left(H_{\mathcal{C}^{\prime}}^{\prime}, H_{\mathcal{B}^{\prime}}^{\prime}\right) \\
\mathcal{H} S\left(H_{\mathcal{B}^{\prime}}^{\prime}, H_{\mathcal{C}^{\prime}}^{\prime}\right) & \mathcal{H} S\left(H_{\mathcal{C}^{\prime}}^{\prime}\right)
\end{array}\right) .
\end{gathered}
$$

We note with respect to the above conclusion that, following [21, Theorem $\mathrm{B}]$ and [9, Theorem 2], $A \oplus A^{o p}$ and $A^{\prime} \oplus\left(A^{\prime}\right)^{o p}$ are, respectively, $\mathbb{Z}_{2}$-graded $\delta$-tight algebra envelopes of $\mathcal{P} \mathcal{J} \mathcal{T}(A)$ and $\mathcal{P} \mathcal{J} \mathcal{T}\left(A^{\prime}\right)$ with $\delta(x, y):=(y, x)$, and that the infinite dimensional nature of the above Jordan triple systems 
forces its hermitian character (following McCrimmons terminology), and so the Zel'manov polynomials in [21, Theorem B] do not vanish on them (see [25, p. 143]).

By an easy argument, we have two possibilities for $F$, either $F(A \oplus\{0\})=$ $A \oplus\{0\}$ or

$$
F(A \oplus\{0\})=\{0\} \oplus A^{o p} .
$$

We have in the first case that $F(x y)=F(x) F(y)$ for any $x, y \in A$, being $L\left(a_{i}\right)=F\left(a_{i}\right)$ for $a_{i} \in A_{-1} \cup A_{1}$ because $F$ extends $L$, and in the second case that $F(x y)=F(y) F(x)$ for any $x, y \in A$, also being $L\left(a_{i}\right)=F\left(a_{i}\right)$ for $a_{i} \in A_{-1} \cup A_{1}$. We note that $F$ is continuous (see $\S 1$ ). This fact, the continuity of $*$ and the equality $A_{0}=\overline{A_{-1} A_{1}+A_{1} A_{-1}}$ give us that $F$ commutes with $*$ and so $F$ is isometric (up to a positive multiple of the inner product) and then $F\left(A_{0}\right)=A_{0}^{\prime}$. Finally, let us show that $F\left(A_{0 j}\right)=L\left(A_{0 j}\right)=A_{0 j}^{\prime}, j \in\{0,1\}$. As $F, L: \mathcal{L T}\left(A_{0}\right) \rightarrow \mathcal{L T}\left(A_{0}^{\prime}\right)$ are isometric (up to a positive multiple of the inner product), $*$-isomorphisms of Lie triple systems, being $A_{01}, A_{01}^{\prime}$ the only infinite dimensional closed ideals of $\mathcal{L} \mathcal{T}\left(A_{0}\right)$ and $\mathcal{L} \mathcal{T}\left(A_{0}^{\prime}\right)$ respectively, then $F\left(A_{01}\right)=L\left(A_{01}\right)=A_{01}^{\prime}$, and therefore $F\left(A_{00}\right)=L\left(A_{00}\right)=A_{00}^{\prime}$.

Taking into account the matrix representation of a topologically simple associative $H^{*}$-algebra, see $\S 1$, we can formulate Theorem 3.1 as follows:

Theorem 3.2. Let $A=\mathcal{M}_{\mathcal{A}}(\mathbb{C})$ be an infinite dimensional topologically simple associative $H^{*}$-algebra and $L: A \rightarrow A$ a Lie triple $*$-automorphism. Then $\mathcal{A}=\mathcal{B} \cup \mathcal{C}$ with $\mathcal{B} \cap \mathcal{C}=\emptyset, \mathcal{B}, \mathcal{C} \neq \emptyset, \mathcal{B}$ of arbitrary finite cardinality, and there exists an automorphism or an anti-automorphism $F$ of $A$ such that $L=F$ on $\mathcal{M}_{\mathcal{B}, \mathcal{C}}(\mathbb{C}) \oplus \mathcal{M}_{\mathcal{C}, \mathcal{B}}(\mathbb{C})$ and $L\left(\mathcal{M}_{\mathcal{B}}(\mathbb{C})\right)=F\left(\mathcal{M}_{\mathcal{B}}(\mathbb{C})\right), L\left(\mathcal{M}_{\mathcal{C}}(\mathbb{C})\right)=F\left(\mathcal{M}_{\mathcal{C}}(\mathbb{C})\right)$.

Let us observe that the mapping $f:=L F^{-1}: A \rightarrow A$ is an automorphism of the Lie triple system $\mathcal{L} \mathcal{T}(A)$ acting as the identity on $\mathcal{M}_{\mathcal{B}, \mathcal{C}}(\mathbb{C}) \oplus \mathcal{M}_{\mathcal{C}, \mathcal{B}}(\mathbb{C})$ and $f\left(\mathcal{M}_{\mathcal{B}}(\mathbb{C})\right)=\mathcal{M}_{\mathcal{B}}(\mathbb{C}), f\left(\mathcal{M}_{\mathcal{C}}(\mathbb{C})\right)=\mathcal{M}_{\mathcal{C}}(\mathbb{C})$. Let us study such a mapping:

Theorem 3.3. Let $A=\mathcal{M}_{\mathcal{A}}(\mathbb{C})$ be an infinite dimensional topologically simple associative $H^{*}$-algebra. Suppose $\mathcal{A}=\mathcal{B} \cup \mathcal{C}$ with $\mathcal{B} \cap \mathcal{C}=\emptyset, \mathcal{B}, \mathcal{C} \neq \emptyset$, and let $f: A \rightarrow A$ be a Lie triple automorphism of $A$ such that $f(x)=x$ for any $x \in \mathcal{M}_{\mathcal{B}, \mathcal{C}}(\mathbb{C}) \oplus \mathcal{M}_{\mathcal{C}, \mathcal{B}}(\mathbb{C})$ and $f\left(\mathcal{M}_{\mathcal{B}}(\mathbb{C})\right)=\mathcal{M}_{\mathcal{B}}(\mathbb{C}), f\left(\mathcal{M}_{\mathcal{C}}(\mathbb{C})\right)=\mathcal{M}_{\mathcal{C}}(\mathbb{C})$. Then $f$ is either the identity map or $f(x)=-x$ for $x \in \mathcal{M}_{\mathcal{B}}(\mathbb{C}) \oplus \mathcal{M}_{\mathcal{C}}(\mathbb{C})$.

Proof. If we denote the elementary matrices in $A=\mathcal{M}_{\mathcal{A}}(\mathbb{C})$ by $e_{i j}$ as usual, then we know that $f\left(e_{i j}\right)=e_{i j}$ for any $(i, j) \in(\mathcal{B} \times \mathcal{C}) \cup(\mathcal{C} \times \mathcal{B})$. Let us compute $f\left(e_{i i}\right)$ and $f\left(e_{k k}\right)$ for $(i, k) \in \mathcal{B} \times \mathcal{C}$ : We can write

$$
f\left(e_{i i}\right)=\sum \lambda_{p q} e_{p q},
$$

where $p, q \in \mathcal{B}$ and

$$
f\left(e_{k k}\right)=\sum \mu_{r s} e_{r s}
$$


with $r, s \in \mathcal{C}, \lambda_{p q}, \mu_{r s} \in \mathbb{C}$. Applying $f$ to the equality $\left[e_{i i}, e_{i k}, e_{k i}\right]=e_{i i}-e_{k k}$ we obtain

$$
\left[\sum \lambda_{p q} e_{p q}, e_{i k}, e_{k i}\right]=\sum \lambda_{p q} e_{p q}-\sum \mu_{r s} e_{r s} .
$$

As we also have

$$
\left[\sum \lambda_{p q} e_{p q}, e_{i k}, e_{k i}\right]=\sum_{p} \lambda_{p i} e_{p i}-\lambda_{i i} e_{k k}
$$

(2) and (3) give us

$$
\sum \lambda_{p q} e_{p q}-\sum \mu_{r s} e_{r s}=\sum_{p} \lambda_{p i} e_{p i}-\lambda_{i i} e_{k k}
$$

and so $\lambda_{p q}=0$ if $q \neq i, \mu_{r s}=0$ if $(r, s) \neq(k, k)$ and $\mu_{k k}=\lambda_{i i}$. As we also have $\left[e_{i i}, e_{k i}, e_{i k}\right]=e_{i i}-e_{k k}$, by applying $f$ as above we obtain

$$
\left[\sum \lambda_{p q} e_{p q}, e_{k i}, e_{i k}\right]=\sum \lambda_{p q} e_{p q}-\sum \mu_{r s} e_{r s},
$$

and as $\left[\sum \lambda_{p q} e_{p q}, e_{k i}, e_{i k}\right]=\sum_{q} \lambda_{i q} e_{i q}-\lambda_{i i} e_{k k}$, we conclude $\sum \lambda_{p q} e_{p q}-\sum \mu_{r s} e_{r s}$ $=\sum_{q} \lambda_{i q} e_{i q}-\lambda_{i i} e_{k k}$ and so $\lambda_{p q}=0$ if $p \neq i$. We have proved $f\left(e_{i i}\right)=\lambda_{i i} e_{i i}$ and $f\left(e_{k k}\right)=\lambda_{i i} e_{k k}$. Moreover, since $\left[e_{i i}, e_{i k}, e_{i i}\right]=-e_{i k}$, we have $\lambda_{i i}^{2}\left[e_{i i}, e_{i k}, e_{i i}\right]=$ $-e_{i k}$ and so $\epsilon:=\lambda_{i i}= \pm 1$. From here, $f\left(e_{i i}\right)=\epsilon e_{i i}$ and $f\left(e_{k k}\right)=\epsilon e_{k k}$ for any $i \in \mathcal{B}$ and $k \in \mathcal{C}$.

Let us compute $f\left(e_{i j}\right)$ with $i, j \in \mathcal{B}, i \neq j$ : We can write

$$
f\left(e_{i j}\right)=\sum \gamma_{p q} e_{p q}
$$

with any $p, q \in \mathcal{B}$. Let us fix $k \in \mathcal{C}$. As $e_{i j}=\left[e_{i j}, e_{j k}, e_{k j}\right]$, we have $\sum \gamma_{p q} e_{p q}=$ $\left[\sum \gamma_{p q} e_{p q}, e_{j k}, e_{k j}\right]=\sum_{p} \gamma_{p j} e_{p j}-\gamma_{j j} e_{k k}$. From here, $\gamma_{p q}=0$ if $q \neq j$ and $\gamma_{j j}=0$. In a similar way, the equality $e_{i j}=\left[e_{i j}, e_{k i}, e_{i k}\right]$ gives us $\sum \gamma_{p q} e_{p q}=$ $\sum_{q} \gamma_{i q} e_{i q}-\gamma_{i i} e_{k k}$ and so $\gamma_{p q}=0$ if $p \neq i$ and $\gamma_{i i}=0$. We conclude $f\left(e_{i j}\right)=$ $\gamma_{i j} e_{i j}$. From $\left[e_{i j}, e_{j k}, e_{k k}\right]=e_{i k}$, we deduce $\gamma_{i j} \epsilon\left[e_{i j}, e_{j k}, e_{k k}\right]=e_{i k}$ and then $\gamma_{i j}=\epsilon$. We obtain a similar result for any $f\left(e_{i j}\right)$ with $i, j \in \mathcal{C}, i \neq j$, and the theorem is proved.

Taking into account the comment after Theorem 3.2, we have the following corollary:

Corollary 3.1. Let $A=\mathcal{M}_{\mathcal{A}}(\mathbb{C})$ be an infinite dimensional topologically simple associative $H^{*}$-algebra, $L: A \rightarrow A$ a Lie triple automorphism of $A$ and $F$ : $A \rightarrow A$ an automorphism or an anti-automorphism of $A$, such that $L=F$ on $\mathcal{M}_{\mathcal{B}, \mathcal{C}}(\mathbb{C}) \oplus \mathcal{M}_{\mathcal{C}, \mathcal{B}}(\mathbb{C})$ and $L\left(\mathcal{M}_{\mathcal{B}}(\mathbb{C})\right)=F\left(\mathcal{M}_{\mathcal{B}}(\mathbb{C})\right), L\left(\mathcal{M}_{\mathcal{C}}(\mathbb{C})\right)=F\left(\mathcal{M}_{\mathcal{C}}(\mathbb{C})\right)$. Then either $L=F$ or $L=f F$ where $f$ is the identity map on $\mathcal{M}_{\mathcal{B}, \mathcal{C}}(\mathbb{C}) \oplus$ $\mathcal{M}_{\mathcal{C}, \mathcal{B}}(\mathbb{C})$ and the negative of the identity on $\mathcal{M}_{\mathcal{B}}(\mathbb{C}) \oplus \mathcal{M}_{\mathcal{C}}(\mathbb{C})$.

We observe that in the second case, as $f$ is the negative of an automorphism, then $L$ is the negative of an automorphism when $F$ is an automorphism, 
and $L$ is the negative of an anti-automorphism in the case that $F$ is an antiautomorphism. This observation together with Theorem 3.2 allow us to claim the following theorem.

Theorem 3.4. Let $L$ be a Lie triple *-automorphism of an infinite dimensional topologically simple associative $H^{*}$-algebra $A$. Then $L$ is either a*automorphism, a*-anti-automorphism, the negative of a*-automorphism or the negative of a*-anti-automorphism of $A$.

Corollary 3.2. Let $L: A \rightarrow A^{\prime}$ be a Lie triple $*$-isomorphism between two infinite dimensional topologically simple associative $H^{*}$-algebras $A, A^{\prime}$. Then $L$ is either $a *$-isomorphism, a*-anti-isomorphism, the negative of a*-isomorphism or the negative of $a *$-anti-isomorphism.

Proof. Since $L: \mathcal{L} \mathcal{T}(A) \rightarrow \mathcal{L} \mathcal{T}\left(A^{\prime}\right)$ is a $*$-isomorphism and by [14] isometric (up to a positive multiple of the inner product), the hilbertian dimensions of $A$ and $A^{\prime}$ agree. So, $A$ and $A^{\prime}$ are $*$-isomorphic to $\mathcal{H} S(H)$, via $\phi, \phi^{\prime}$ respectively. If we write by $L^{\prime}$ the only mapping making commutative the below diagram, the commutativity of the diagram joint with Theorem 3.4 complete the proof.

$$
\begin{array}{ccccc} 
& & & L & \\
& A & \rightarrow & A^{\prime} & \\
\phi & \downarrow & & \downarrow & \phi^{\prime} \\
& \mathcal{H} S(H) & \rightarrow & \mathcal{H} S(H) & \\
& & L^{\prime} & &
\end{array}
$$

Corollary 3.3. Let $L: A \rightarrow A^{\prime}$ be a Lie triple isomorphism between two infinite dimensional topologically simple associative $H^{*}$-algebras $A, A^{\prime}$. Then $L$ is either an isomorphism, an anti-isomorphism, the negative of an isomorphism or the negative of an anti-isomorphism.

Proof. Consider in $A^{\prime}$ the unique $L^{*}$-triple structure making $L: \mathcal{L} \mathcal{T}(A) \rightarrow$ $\mathcal{L} \mathcal{T}\left(A^{\prime}\right)$ an isometric $*$-isomorphism (of topologically simple $L^{*}$-triples). As $A \perp A$ is a $\mathbb{Z}_{2}$-graded $L^{*}$-algebra envelope of $\mathcal{L} \mathcal{T}(A)$, then the $\mathbb{Z}_{2}$-graded $L^{*}$ algebra envelope of $A^{\prime}$ is of the type $B \perp B$ with $B$ a topologically simple associative $H^{*}$-algebra, and being $B \perp B *$-isometrically isomorphic to $A \perp A$ (see [10, Proposition 2.1]). From here, $L: A \rightarrow B$, where $B$ is $A^{\prime}$ considered with its new involution and inner product, is a Lie triple $*$-isomorphism between two infinite dimensional topologically simple associative $H^{*}$-algebras. By Corollary 3.2, $L$ is either a $*$-isomorphism, a $*$-anti-isomorphism, the negative of a $*$-isomorphism or the negative of a $*$-anti-isomorphism. The result follows from here.

\subsection{The finite dimensional case}

The infinite dimension of the polarized Jordan triple systems $\mathcal{P} \mathcal{J} \mathcal{T}(A)$ and $\mathcal{P} \mathcal{J} \mathcal{T}(A)^{\prime}$ in Theorem 3.1 is needed to apply the key D'Amour's extension 
results [21, Theorem B]. Therefore, we will develop new arguments to study the finite dimensional case.

As it is well-known, any topologically simple associative $H^{*}$-algebra $A$ with finite dimension $n>1$ is isomorphic to an algebra of the type $\mathcal{M}_{n}(\mathbb{C})$.

Lemma 3.1. If we write $A=\mathcal{M}_{n}(\mathbb{C})=\mathbb{C} I d \oplus\left[\mathcal{M}_{n}(\mathbb{C}), \mathcal{M}_{n}(\mathbb{C})\right]$ and denote by $L$ a Lie triple automorphism of $A$. Then $L(\mathbb{C} I d)=\mathbb{C} I d$ and

$$
L\left(\left[\mathcal{M}_{n}(\mathbb{C}), \mathcal{M}_{n}(\mathbb{C})\right]\right)=\left[\mathcal{M}_{n}(\mathbb{C}), \mathcal{M}_{n}(\mathbb{C})\right]
$$

Proof. Let us write by $T$ the Lie triple system $\mathcal{L} \mathcal{T} \mathcal{S}(A)$. It is easy to verify that $\operatorname{Ann}(T)=\mathbb{C} I d$. Indeed, clearly $\mathbb{C} I d \subset \operatorname{Ann}(T)$ and conversely, given $x \in T$ such that $[x, a, b]=[[x, a], b]=0$ for any $a, b \in T$, we have $[x, a]=\lambda I d$, with $\lambda \in \mathbb{C}$. From here, $[x, a] \in \mathbb{C} I d \cap \operatorname{sl}(n, \mathbb{C})=0$, then $[x, a]=0$ for all $a \in T$ and so $x \in \mathbb{C} I d$. As $L$ is a Lie triple automorphism of $T$, we conclude $L(\mathbb{C} I d)=\mathbb{C} I d$. Since $A_{n-1}=\left[\mathcal{M}_{n}(\mathbb{C}), \mathcal{M}_{n}(\mathbb{C})\right]$ is the only nonzero minimal ideal of $T$, then $L\left(\left[\mathcal{M}_{n}(\mathbb{C}), \mathcal{M}_{n}(\mathbb{C})\right]\right)=\left[\mathcal{M}_{n}(\mathbb{C}), \mathcal{M}_{n}(\mathbb{C})\right]$.

So let $\left.L\right|_{A_{l}}: A_{l} \rightarrow A_{l}$ be a Lie triple automorphism and consider the well defined map $G: A_{l} \rightarrow A_{l}$ such that $G\left(\sum_{i}\left[x_{i}, y_{i}\right]\right)=\sum_{i}\left[L\left(x_{i}\right), L\left(y_{i}\right)\right]$ for $x_{i}, y_{i} \in A_{l}$. For convenience we will write $L:=\left.L\right|_{A_{l}}$. Next we prove the identity $G([[a, b],[c, d]])=[G([a, b]), G([c, d])]$ for $a, b, c, d \in A_{l}$, by using the fact $[[a, b],[c, d]]=-[[b,[c, d]], a]-[[[c, d], a], b]$. Thus

$$
\begin{aligned}
G([[a, b],[c, d]]) & =-[[L(b),[L(c), L(d)]], L(a)]-[[[L(c), L(d)], L(a)], L(b)] \\
& =[[L(a), L(b)],[L(c), L(d)]] \\
& =[G([a, b]), G([c, d])],
\end{aligned}
$$

which proves that $G$ is a Lie algebra automorphism of $A_{l}$. On the other hand we also have

$$
L([x, y])=[G(x), L(y)]=[L(x), G(y)]
$$

for $x, y \in A_{l}$, since $x=\sum_{i}\left[a_{i}, b_{i}\right]$ and

$$
\begin{aligned}
L([x, y]) & =\sum_{i} L\left(\left[\left[a_{i}, b_{i}\right], y\right]\right)=\sum_{i}\left[\left[L\left(a_{i}\right), L\left(b_{i}\right)\right], L(y)\right] \\
& =\left[G\left(\sum_{i}\left[a_{i}, b_{i}\right]\right), L(y)\right]=[G(x), L(y)] .
\end{aligned}
$$

In a similar way it is proved that $L([x, y])=[L(x), G(y)]$. Thus we have proved the set of equalities:

$$
\begin{aligned}
G([x, y]) & =[G(x), G(y)]=[L(x), L(y)], \\
L([x, y]) & =[L(x), G(y)]=[G(x), L(y)] .
\end{aligned}
$$

Lemma 3.2. We have $\left(L^{-1} G\right)^{2}=\left(G L^{-1}\right)^{2}=1$.

Proof. As

$$
[L(x), G(y)]=\left[L(x), L\left(L^{-1} G(y)\right)\right]=\left[G(x), G L^{-1} G(y)\right]
$$




$$
=\left[G(x), L L^{-1} G L^{-1} G(y)\right]=\left[L(x), G L^{-1} G L^{-1} G(y)\right],
$$

we deduce $G(y)=G L^{-1} G L^{-1} G(y)$ and so $L^{-1} G L^{-1} G=1, G L^{-1} G L^{-1}=1$. The lemma is proved.

Since $\left(L^{-1} G\right)^{2}=1$ we can decompose $A_{l}$ as the direct sum of two subspaces so that $L^{-1} G$ acts as the identity on one of the direct summands while it acts as the negative of the identity on the other summand. Thus $L$ and $G$ agree on one of the summands and $L=-G$ on the other one. Next we show that the subspace on which $L$ and $G$ agree is a Lie triple ideal of $A_{l}$ so that given the simplicity of $A_{l}$ as a Lie triple, this subspace is 0 or the whole $A_{l}$.

Corollary 3.4. $L= \pm G$.

Proof. For $\epsilon= \pm 1$ write $T_{\epsilon}=\left\{x \in A_{l}: L(x)=\epsilon G(x)\right\}$ so that $A_{l}=T_{1} \oplus T_{-1}$. It is immediate that $\left[T_{\alpha}, T_{\beta}, T_{\gamma}\right] \subset T_{\alpha \beta \gamma}$ for $\alpha, \beta, \gamma \in\{-1,1\}$. But taking $x \in T_{1}$ and $y \in T_{-1}$ we have $[L(x), G(y)]=[G(x),-L(y)]$ which compared with (5) gives $[L(x), G(y)]=0$, that is, $\left[L\left(T_{1}\right), G\left(T_{-1}\right)\right]=\left[L\left(T_{1}\right), L\left(T_{-1}\right)\right]=0$. Hence $\left[L\left(T_{1}\right), L\left(T_{-1}\right), L(T)\right]=0$ implying $\left[T_{1}, T_{-1}, T\right]=0$, that is, $\left[T_{1}, T_{-1}\right]=0$. Also $\left[T_{1}, T_{1}, T_{-1}\right]=0$ applying Jacobi identity. Thus $\left[T_{1}, T, T\right]=\left[T_{1}, T_{1}, T\right]=$ $\left[T_{1}, T_{1}, T_{1}\right] \subset T_{1}$ which proves that $T_{1}$ is an ideal of the triple system $A_{l}$. The simplicity of this Lie triple implies that $T_{1}=0$ (implying $L=-G$ ) or $T_{1}=A_{l}$ (hence $L=G$ ).

Theorem 3.5. Let $L: \mathcal{M}_{n}(\mathbb{C}) \rightarrow \mathcal{M}_{n}(\mathbb{C}), n>1$, be a Lie triple automorphism. Then there exists an automorphism, an anti-automorphism, the negative of an automorphism or the negative of an anti-automorphism $F$ : $\mathcal{M}_{n}(\mathbb{C}) \rightarrow \mathcal{M}_{n}(\mathbb{C})$ such that $\delta:=L-F$ is a linear map from $\mathcal{M}_{n}(\mathbb{C})$ onto its center sending commutators to zero.

Proof. By Corollary 3.4 we have two possibilities for $\left.L\right|_{A_{l}}$. In the first one, $\left.L\right|_{A_{l}}$ is an automorphism of the Lie algebra $A_{l}$. By applying [22, Theorem 5, p. 283], $\left.L\right|_{A_{l}}: A_{l} \rightarrow A_{l}$ extends to an automorphism or the negative of an anti-automorphism $F: \mathcal{M}_{n}(\mathbb{C}) \rightarrow \mathcal{M}_{n}(\mathbb{C})$. In the second one, $-\left.L\right|_{A_{l}}$ is an automorphism of the Lie algebra $A_{l}$. [22, Theorem 5, p. 283] gives us now that $-\left.L\right|_{A_{l}}$ extends to an automorphism or the negative of an anti-automorphism $F^{\prime}$ of $\mathcal{M}_{n}(\mathbb{C})$. In this case $\left.L\right|_{A_{l}}$ extends to $F:=-F^{\prime}: \mathcal{M}_{n}(\mathbb{C}) \rightarrow \mathcal{M}_{n}(\mathbb{C})$, $F$ being the negative of an automorphism or an anti-automorphism. If we call $\delta:=L-F$, we can prove that $\delta\left(\mathcal{M}_{n}(\mathbb{C})\right) \subset Z\left(\mathcal{M}_{n}(\mathbb{C})\right)=\mathbb{C} I d$ and $\delta\left(\left[\mathcal{M}_{n}(\mathbb{C}), \mathcal{M}_{n}(\mathbb{C})\right]\right)=0$ : Given any $x \in \mathcal{M}_{n}(\mathbb{C}), x=c+a$ with $c \in \mathbb{C} I d$ and $a \in\left[\mathcal{M}_{n}(\mathbb{C}), \mathcal{M}_{n}(\mathbb{C})\right]$. The character of automorphism, anti-automorphism, negative of an automorphism or negative of an anti-automorphism of $F$ gives us $F(c) \in \mathbb{C} I d$. By Lemma 3.1, we also have $L(c) \in \mathbb{C} I d$. Finally, as $F(a)=L(a)$ for any $a \in\left[\mathcal{M}_{n}(\mathbb{C}), \mathcal{M}_{n}(\mathbb{C})\right]$, we conclude $\delta\left(\mathcal{M}_{n}(\mathbb{C})\right) \subset \mathbb{C} I d$ and

$$
\delta\left(\left[\mathcal{M}_{n}(\mathbb{C}), \mathcal{M}_{n}(\mathbb{C})\right]\right)=0 .
$$




\subsection{The annihilator zero case}

Theorem 3.6. Let $L$ be a continuous Lie triple automorphism of an associative $H^{*}$-algebra with zero annihilator $A$. Then there exist closed ideals $P, Q, R, S$ of $A$ and $a \mathbb{C}$-linear bijective mapping $F: A \rightarrow A$ such that $A=P \perp Q \perp R \perp S$, and if we denote by $\left\{I_{\alpha}\right\}$ the family of the minimal closed ideals of $A$, then:

(1) F restricted to $P$ is an isomorphism.

(2) F restricted to $Q$ is an anti-isomorphism.

(3) $F$ restricted to $R$ is the negative of an isomorphism.

(4) F restricted to $S$ is the negative of an anti-isomorphism.

(5) If $I_{\alpha}$ is infinite dimensional, then $\left.F\right|_{I_{\alpha}}=\left.L\right|_{I_{\alpha}}$.

(6) If $I_{\alpha}$ is finite dimensional, then $\delta_{\alpha}:=\left.L\right|_{I_{\alpha}}-\left.F\right|_{I_{\alpha}}$ is a linear mapping from $I_{\alpha}$ onto the center of $A$ sending commutators to zero.

Proof. Denote by $\left\{I_{\alpha}\right\}_{\alpha \in \Lambda}$ the family of minimal closed ideals of $A$. Let us consider $I_{\alpha_{0}} \in\left\{I_{\alpha}\right\}_{\alpha \in \Lambda}$.

If $I_{\alpha_{0}}$ is infinite dimensional, as $I_{\alpha_{0}}$ is a topologically simple $L^{*}$-triple with $\mathbb{Z}_{2}$-graded $L^{*}$-algebra envelope $I_{\alpha_{0}} \perp I_{\alpha_{0}}$, then $L^{-1}\left(I_{\alpha_{0}}\right)$ is also a topologically simple $L^{*}$-triple with a $\mathbb{Z}_{2}$-graded $L^{*}$-algebra envelope of the type $L^{-1}\left(I_{\alpha_{0}}\right) \perp$ $L^{-1}\left(I_{\alpha_{0}}\right), L^{-1}\left(I_{\alpha_{0}}\right)$ being an infinite dimensional minimal closed ideal of $A$. If we denote by $F_{\alpha_{0}}$ the restriction of $L$ to $L^{-1}\left(I_{\alpha_{0}}\right)$, Corollary 3.3 shows that $F_{\alpha_{0}}$ is either an isomorphism, an anti-isomorphism, a negative of an isomorphism or a negative of an anti-isomorphism.

If $I_{\alpha_{0}}$ is finite dimensional with $\operatorname{dim} I_{\alpha_{0}}>1$, as $I_{\alpha_{0}}$ is isomorphic to an associative algebra of the type $\mathcal{M}_{n}(\mathbb{C}), n>1$, then we have as in Lemma 3.1 that $L^{-1}\left(I_{\alpha_{0}}\right)$ is also a minimal ideal of $A$ isomorphic to $\mathcal{M}_{n}(\mathbb{C})$. If we denote by $L_{\alpha_{0}}$ the restriction of $L$ to $L^{-1}\left(I_{\alpha_{0}}\right)$, Theorem 3.5 gives us that there exists an automorphism, an anti-automorphism, a negative of an automorphism or a negative of an anti-automorphism $F_{\alpha_{0}}: L^{-1}\left(I_{\alpha_{0}}\right) \rightarrow I_{\alpha_{0}}$ such that $\delta_{\alpha_{0}}:=L_{\alpha_{0}}-$ $F_{\alpha_{0}}$ is a linear map from $L^{-1}\left(I_{\alpha_{0}}\right)$ onto the center of $I_{\alpha_{0}}$ sending commutators to zero.

Let $I_{\alpha_{0}}$ be such that $\operatorname{dim} I_{\alpha_{0}}=1$. Since we have the family of linear isomorphisms $\left\{F_{\beta}\right\}, F_{\beta}: L_{\beta_{0}}^{-1}\left(I_{\beta}\right) \rightarrow I_{\beta}$ among the minimal closed ideals of dimension not 1 , we define the unique linear isomorphism $F_{\alpha_{0}}: I_{\alpha_{0}} \rightarrow I_{\alpha_{0}}$ given by $F_{\alpha_{0}}(1)=1$ which turns out to be an automorphism (of associative algebras).

Let consider any $I_{\alpha} \in\left\{I_{\alpha}\right\}_{\alpha \in \Lambda}$ with the unique $H^{*}$-structure that makes $F_{\alpha}$ either a $*$-isometric isomorphism, a $*$-isometric anti-isomorphism, a $*$-isometric negative of an isomorphism or a $*$-isometric negative of an anti-isomorphism. As $A=\overline{\perp_{\alpha \in \Lambda} I_{\alpha}}$, the isometric character of any $F_{\alpha}, \alpha \in \Lambda$, enable us to extend $\left\{F_{\alpha}\right\}_{\alpha \in \Lambda}$ to an isometric linear mapping $F: A \rightarrow A$ such that

$$
A=\left(\overline{\perp_{\alpha \in \Lambda_{1}} I_{\alpha}}\right) \perp\left(\overline{\perp_{\alpha \in \Lambda_{2}} I_{\alpha}}\right) \perp\left(\overline{\perp_{\alpha \in \Lambda_{3}} I_{\alpha}}\right) \perp\left(\overline{\perp_{\alpha \in \Lambda_{4}} I_{\alpha}}\right),
$$

with $\Lambda_{1} \cup \Lambda_{2} \cup \Lambda_{3} \cup \Lambda_{4}=\Lambda, \Lambda_{i} \cap \Lambda_{j}=\emptyset$ for $i \neq j$, and being $F$ restricted to $P:=\overline{\perp_{\alpha \in \Lambda_{1}} I_{\alpha}}$ an isomorphism, $F$ restricted to $Q:=\overline{\perp_{\alpha \in \Lambda_{2}} I_{\alpha}}$ an antiisomorphism, $F$ restricted to $R:=\overline{\perp_{\alpha \in \Lambda_{3}} I_{\alpha}}$ a negative of an automorphism, 
and $F$ restricted to $S:=\overline{\perp_{\alpha \in \Lambda_{4}} I_{\alpha}}$ a negative of an anti-isomorphism. It is clear that $P, Q, R, S$ and $F$ satisfy the conditions of Theorem 3.6.

\section{References}

[1] W. Ambrose, Structure theorems for a special class of Banach algebras, Trans. Amer. Math. Soc. 57 (1945), 364-386.

[2] R. Banning and M. Mathieu, Commutativity preserving mappings on semiprime rings, Comm. Algebra 25 (1997), no. 1, 247-265.

[3] K. I. Beidar, M. Brešar, M. A. Chebotar, and W. S. Martindale, 3rd On Herstein's Lie map conjectures. III, J. Algebra 249 (2002), no. 1, 59-94.

[4] K. I. Beidar, W. S. Martindale, and A. V. Mikhalëv, Lie isomorphisms in prime rings with involution, J. Algebra 169 (1994), no. 1, 304-327.

[5] M. I. Berenguer and A. R. Villena, Continuity of Lie isomorphisms of Banach algebras, Bull. London Math. Soc. 31 (1999), no. 1, 6-10.

[6] M. Brešar, Commuting traces of biadditive mappings, commutativity-preserving mappings and Lie mappings, Trans. Amer. Math. Soc. 335 (1993), no. 2, 525-546.

[7] _ Functional identities: a survey, Algebra and its applications (Athens, OH, 1999), 93-109, Contemp. Math., 259, Amer. Math. Soc., Providence, RI, 2000.

[8] M. Cabrera, J. Martínez, and A. Rodríguez, Structurable $H^{*}$-algebras, J. Algebra 147 (1992), no. 1, 19-62.

[9] A. J. Calderón Martín and C. Martín González, Dual pairs techniques in $H^{*}$-theories, J. Pure Appl. Algebra 133 (1998), no. 1-2, 59-63.

[10] Hilbert space methods in the theory of Lie triple systems, Recent progress in functional analysis (Valencia, 2000), 309-319, North-Holland Math. Stud., 189, NorthHolland, Amsterdam, 2001.

[11] _ Lie isomorphisms on $H^{*}$-algebras, Comm. Algebra 31 (2003), no. 1, 333-343.

[12] A structure theory for Jordan $H^{*}$-pairs, Boll. Unione Mat. Ital. Sez. B Artic. Ric. Mat. (8) 7 (2004), no. 1, 61-77.

[13] ture Notes in Pure and Applied Math. Marcel Dekker Inc. (2004), 87-94.

[14] A. Castellón Serrano and J. A. Cuenca Mira, Isomorphisms of $H^{*}$-triple systems, Ann. Scuola Norm. Sup. Pisa Cl. Sci. (4) 19 (1992), no. 4, 507-514.

[15] A. Castellón Serrano, J. A. Cuenca Mira, and C. Martín González, Ternary H*-algebras, Boll. Un. Mat. Ital. B (7) 6 (1992), no. 1, 217-228.

[16] M. A. Chebotar, On Lie automorphisms of simple rings of characteristic 2, Fundam. Prikl. Mat. 2 (1996), no. 4, 1257-1268.

[17] _ On Lie isomorphisms in prime rings with involution, Comm. Algebra 27 (1999), no. 6, 2767-2777.

[18] J. A. Cuenca Mira, A. García, and C. Martín González, Structure theory for L*-algebras, Math. Proc. Cambridge Philos. Soc. 107 (1990), no. 2, 361-365.

[19] J. A. Cuenca Mira and Á. Rodríguez, Isomorphisms of $H^{*}$-algebras, Math. Proc. Cambridge Philos. Soc. 97 (1985), no. 1, 93-99.

[20] _ Structure theory for noncommutative Jordan $H^{*}$-algebras, J. Algebra 106 (1987), no. 1, 1-14.

[21] A. D'Amour, Jordan triple homomorphisms of associative structures, Comm. Algebra 19 (1991), no. 4, 1229-1247.

[22] N. Jacobson, Lie Algebras, Interscience Tracts in Pure and Applied Mathematics, No. 10 Interscience Publishers (a division of John Wiley \& Sons), New York-London, 1962.

[23] _ Structure of Rings, American Mathematical Society, Colloquium Publications, vol. 37. American Mathematical Society, 190 Hope Street, Prov., R. I., 1956. 
[24] P. Ji and L. Wang, Lie triple derivations of TUHF algebras, Linear Algebra Appl. 403 (2005), 399-408.

[25] K. McCrimmon and E. Zel'manov, The structure of strongly prime quadratic Jordan algebras, Adv. in Math. 69 (1988), no. 2, 133-222.

[26] M. Mathieu, Lie mappings of $C^{*}$-algebras, Nonassociative algebra and its applications (Sao Paulo, 1998), 229-234, Lecture Notes in Pure and Appl. Math., 211, Dekker, New York, 2000.

[27] C. R. Miers, Lie *-triple homomorphisms into von Neumann algebras, Proc. Amer. Math. Soc. 58 (1976), 169-172.

[28] E. Neher, On the classification of Lie and Jordan triple systems, Comm. Algebra 13 (1985), no. 12, 2615-2667.

[29] J. R. Schue, Hilbert space methods in the theory of Lie algebras, Trans. Amer. Math. Soc. 95 (1960), 69-80.

[30] J.-H. Zhang, B.-W. Wu, and H.-X. Cao, Lie triple derivations of nest algebras, Linear Algebra Appl. 416 (2006), no. 2-3, 559-567.

A. J. Calderón Martín

Departamento de Matemáticas

Universidad de CÁdiz

11510 Puerto Real, Cádiz, Spain

E-mail address: ajesus.calderon@uca.es

C. Martín GonzÁlez

Departamento de Álgebra

GeOMETRÍA Y TOPOLOGÍA

UNIVERSIDAD DE MÁlaga

APARTADO 59, 29080

MÁlaga, SpAin

E-mail address: candido@apncs.cie.uma.es 